\title{
Study of fluid dynamics at the boundary wall of a microchannel by Bloch surface waves
}

\author{
A. Occhicone, ${ }^{1,}{ }^{*}$ A. Sinibaldi, ${ }^{1}$ F. SonntaG ${ }^{2}$, P. Munzert ${ }^{3}$, N. Danz ${ }^{3}$, And \\ F. MICHELOTTI ${ }^{1}$ \\ ${ }^{1}$ Department of Basic and Applied Sciences for Engineering, SAPIENZA Università di Roma, Roma 00161, Italy \\ ${ }^{2}$ Fraunhofer Institute for Material and Beam Technology IWS, Winterbergstr. 28, Dresden, Germany \\ ${ }^{3}$ Fraunhofer Institute for Applied Optics and Precision Engineering, A. -Einstein-Str. 7, Jena, Germany \\ *e-mail: agostino.occhicone@uniroma1.it
}

Received XX Month XXXX; revised XX Month, XXXX; accepted XX Month XXXX; posted XX Month XXXX (Doc. ID XXXXX); published XX Month XXXX

\begin{abstract}
Understanding how a fluid flows at the boundaries when it is confined at the micro/nano scale is crucial for a broad range of engineering and biology applications. We propose an experimental technique based on Bloch surface waves sustained by a onedimensional photonic crystal to evaluate the speed of the contact line, i.e. the triple junction separating three phases, in the low Reynold's number regime and with a nanometric resolution. Here, we report on the experimental characterization of the translatory motion of the contact line that separates two water solutions with a relatively high refractive index mismatch $\left(7.35 \times 10^{-3}\right)$ and its slipping over a solid surface. The advantages are the relative simplicity and economy of the experimental configuration. (C) 2019 Optical Society of America
\end{abstract}

OCIS codes: (100.0100) Image Processing; (240.6690) Surface waves; (280.4788) Optical sensing and sensors; (240.0240) Optics at surfaces.

http://dx.doi.org/10.1364/OL.99.099999

The research area of microfluidics emerged due to the appearance of microtechnology in the early '90s [1]. Subsequently, the field rapidly developed in several research fields, i.e. in chemistry, medicine, biology, and so on, with substantial advantages in terms of integration and fabrication costs. Consequently, the demand for new and reliable detection systems increases, therefore making the peculiar nature of surface electromagnetic waves (SEW) very attractive and suitable to characterize fluid flow and mass transport in proximity of boundaries at the micro/nanoscale $[2,3]$. Among all other types of SEW, Bloch surface waves (BSW) are guided at the boundary between a truncated onedimensional photonic crystal (1DPC) and an external homogeneous dielectric medium [4]. 1DPC are dielectric multilayered stacks characterized by a photonic band structure that shows a photonic band gap where light cannot propagate inside the 1DPC. BSWs are localized at the boundary due to Bragg and total internal reflection (TIR) at the 1DPC and homogeneous medium sides, respectively $[5,6]$. Their field envelope decays exponentially both inside the
1DPC and the external medium. Given their extreme sensitivity to any refractive index perturbation at the 1DPC/external medium interface, BSW were demonstrated to provide the accuracy and the dynamic range that are needed for biosensing applications [7]. Moreover, BSW were used for early cancer biomarkers detection [8], enhanced Goos-Hänchen shift [9], surface-enhanced Raman scattering [10], room temperature polaritons [11], and surface integrated optics [12].

Here, we report on the application of a BSW based sensing scheme to probe fluid flow in close proximity of a flat solid boundary, as guaranteed by the evanescent character of the BSW. The proposed technique could complement welldeveloped techniques based on either particle-tracking velocimetry [13] or particle image velocimetry (PIV) [14], particularly tomographic PIV [15] or $\mu$-PIV [16], by suppling information otherwise not available.

The 1DPCs used in the present work were fabricated by evaporating under high vacuum conditions $\mathrm{SiO}_{2}, \mathrm{Ta}_{2} \mathrm{O}_{5}$, and $\mathrm{TiO}_{2}$ layers on standard microscope slides by means of a plasma ion assisted system. The surface roughness of the stack, as measured by atomic force microscopy, was $1.32 \mathrm{~nm}$ (rms). Details on the design and fabrication are given elsewhere [17]. In Fig. 1(a), we sketch the 1DPC, which is constituted by a periodic stack, with thicknesses $\mathrm{d}_{\mathrm{SiO}_{2}}=275 \mathrm{~nm}$ and $\mathrm{d}_{\mathrm{Ta}_{2} \mathrm{O}_{5}}=120 \mathrm{~nm}$, that is topped by two $20 \mathrm{~nm}$ thick layers made out of $\mathrm{TiO}_{2}$ and $\mathrm{SiO}_{2}$, respectively. The refractive indices of $\mathrm{SiO}_{2}, \mathrm{Ta}_{2} \mathrm{O}_{5}$, and $\mathrm{TiO}_{2}$ at $\lambda_{0}=632.8 \mathrm{~nm}$ are: $\quad \mathrm{n}_{\mathrm{SiO}_{2}}=1.447+\mathrm{i} 5 \times 10^{-6}, \quad \mathrm{n}_{\mathrm{Ta}_{2} \mathrm{O}_{5}}=2.075+\mathrm{i} 5 \times 10^{-5}$, $\mathrm{n}_{\mathrm{TiO}_{2}}=2.293+\mathrm{i} 1.83 \times 10^{-3}$. The main advantage of BSW sustained by 1DPC is the possibility to optimize the 1DPC geometry for specific applications [18,19]. The 1DPC were designed for an aqueous external medium [6,20]. In Fig. 1(a), we show the normalized $|\mathrm{E}|^{2}$ field profile of the s-polarized BSW, which is characterized by an evanescent tail penetrating inside the fluid:

$$
|E|^{2}=A e^{-z / L_{p}}
$$

where $L_{P}=102 \mathrm{~nm}$ at $\lambda_{0}, A=0.81$ is the value of the normalized $|\mathrm{E}|^{2}$ at the $1 \mathrm{DPC} /$ fluid interface and $z$ is the orthogonal direction. The intensity profile was calculated by means of the transfer matrix method [21]. Fig. 1(a) shows that 
the evanescent tail can be used to probe the material transport of a fluid flow in close proximity $\left(L_{P}\right)$ of the 1DPC/fluid interface.

In Fig. 1(b), we show the experimental setup used to excite the BSW. A He-Ne laser at $\lambda_{0}$ is collimated and polarized along the TE direction by means of a polarizer. The beam is expanded by means of a telescope constituted by a $40 \times$ microscope objective and a spherical lens (SL, $\mathrm{f}_{1}=250 \mathrm{~mm}$ ); a rotating diffuser inside the telescope destroys the beam spatial coherence. After an iris, the $1 \mathrm{~cm}$ wide beam is focused into a BK7 coupling prism above TIR by a cylindrical lens (CL1, $\mathrm{f}_{2}=150 \mathrm{~mm}$ ), achieving the Kretschmann-Raether TIR configuration (KR) [22]. Alternatively, one could use diffracting elements embedded in the 1DPC in order to get grating coupling to the BSW. Diffracting structures placed at the surface of the 1DPC $[23,24]$, would permit both to couple to the BSW and to study their effect on the fluid flow. A double rotation stage allows to set the average incidence angle $(\theta)$ around the BSW resonance angular position at $\lambda_{0}$ and to collect the reflected beam at $2 \theta$ with a cylindrical lens (CL2, $\mathrm{f}_{3}=150 \mathrm{~mm}$ ), which acts as a Fourier lens that images the angular reflection pattern on a CMOS array detector (Thorlabs DCC1645C, 1280×1024 pixel, 8 bit).
As shown in Fig. 1(c), the back face of the microscope slide is coupled to the BK7 prism by means of an index-matching oil. The front face of the microscope slides is topped with a PDMS flow cell, which permits to bring a fluid in contact with the 1DPC surface. The geometry of the PDMS micro-channel is shown in Fig. 1(d). The channel is $\mathrm{w}=1 \mathrm{~mm}$ wide; the central part of the channel is $17 \mathrm{~mm}$ long; the inlet and the outlet arms of the channels are $13.6 \mathrm{~mm}$ long. The channel height is $\mathrm{h}=200 \mu \mathrm{m}$ and the cross-section $\Sigma=0.2 \mathrm{~mm}^{2}$. The fluidic chip is directly glued onto the 1DPC.

As shown in Fig. 1(c) and Fig. 1(d), the cylindrical focusing and Fourier imaging lenses allow to illuminate a sharp line at the 1DPC surface perpendicularly to the incidence plane and along the micro-channel, i.e. parallel to the fluid flow. Such a configuration permits to sense refractive index changes simultaneously and in parallel along such a line [18]. In Fig. $1(\mathrm{e})$, we show a typical image acquired by the CMOS camera. Every abscissa point corresponds to a different $\theta$ (1280 pixel, $\Delta \theta=1 \mathrm{deg}, \mathrm{c}_{\theta}=0.78 \mathrm{mdeg} / \mathrm{pix}$ ) and every ordinate point to the position $\mathrm{x}$ along the micro-channel (1024 pixel, $\left.\mathrm{l}_{\mathrm{H}}=3.69 \mathrm{~mm}, \mathrm{c}_{\mathrm{l}}=3.6 \mu \mathrm{m} / \mathrm{pix}\right)$. The excitation of a BSW shows up as a resonant dip in the angular reflectance spectrum. The angular position of the dip may vary along $x$ due to local inhomogeneities of the 1DPC and of the fluid.
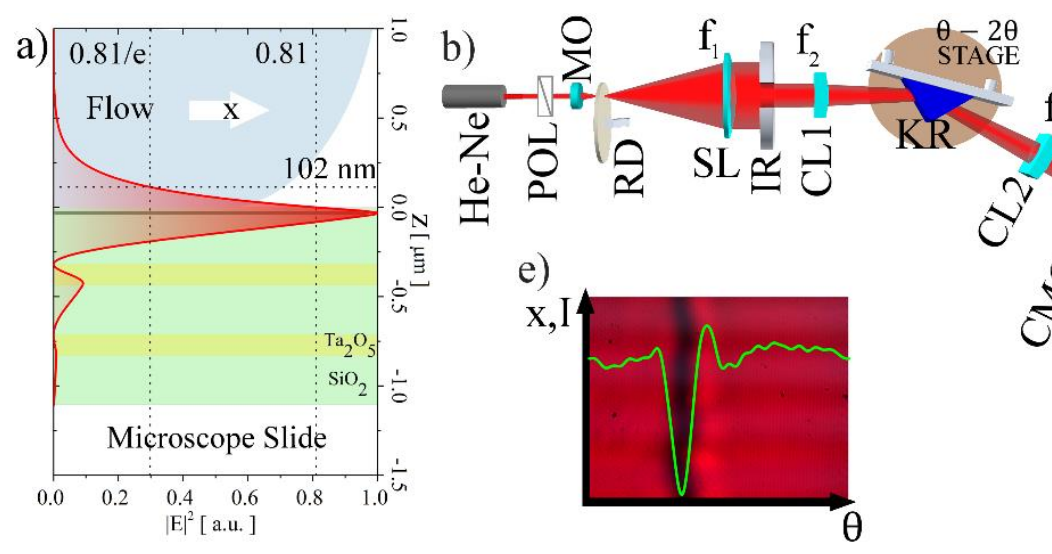

c)

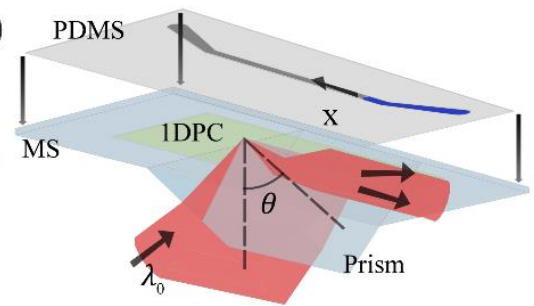

d)

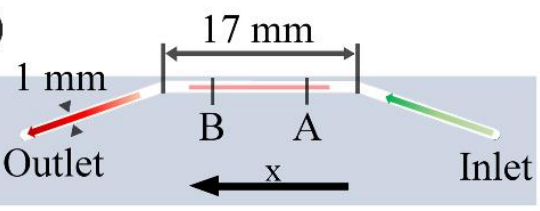

Fig. 1. a) Calculated electric field square modulus in the 1DPC when a BSW is excited. The complete multi-layered geometry is shown and the fluid flow on the top is sketched by a parabolic profile. b) Optical set-up for the excitation of BSW on 1DPC in the KR TIR configuration (Polarizer $\rightarrow$ POL, $40 \times$ microscope objective $\rightarrow$ MO, Rotating Diffuser $\rightarrow$ RD, Iris $\rightarrow$ IR). c) Sketch of the microscope slide with the 1DPC and the BK7 prism in contact through an oil. The 1DPC is topped by the PDMS micro-fluidic channel. d) Fluidic cell geometry. The cell height is $120 \mu \mathrm{m}$, width and length are quoted in the figure. The red line shows where the light is focused. e) Image acquired by the CMOS camera; the green line represents the reflectance, I, in arbitrary units measured along the row 512 .

Here, as an external fluid we used either deionized water or a $5 \%$ glucose solution in deionized water, corresponding to a refractive index contrast $\Delta \mathrm{n}_{\mathrm{MAX}}=7.35 \times 10^{-3}$. In every experiment, the micro-channel is filled with the glucose solution at the beginning and then deionized water is injected by means of a motorized syringe ( 3 port Cavro Pump from Tecan) at a controlled flow rate $\Phi$ until complete substitution of the fluid takes place. During the transition, the CMOS camera acquires the reflected signal at its maximum frame rate of $\mathrm{f}_{\text {sam }}=25 \mathrm{fps}$. Camera acquisition starts when pumping is switched on. The experiments were conducted for six different flow rates, $\Phi: 4.058 \mu \mathrm{L} / \mathrm{s}, 3.162 \mu \mathrm{L} / \mathrm{s}$, $2.266 \mu \mathrm{L} / \mathrm{s}, 1.370 \mu \mathrm{L} / \mathrm{s}, 0.474 \mu \mathrm{L} / \mathrm{s}$ and $0.365 \mu \mathrm{L} / \mathrm{s}$, leading to corresponding Reynolds numbers [25] $R e=\frac{2 \Phi \rho}{\mu(h+w)}$ always lower than 10, where $\rho$ is the specific mass and $\mu=8.9 \cdot 10^{-4} \mathrm{~Pa} \cdot \mathrm{s}$ the dynamic viscosity of water at ambient temperature.

From the experiments, we obtained reflectance videos saved in AVI format. The videos were analyzed frame by frame and the refractive index change was retrieved as a function of time, by means of a proprietary LabView VI and according to the following procedure.

Each frame $f$, as shown in Fig. 1(e), is an 8-bit array of pixels $\left[I_{i j}\right]_{f}$. The plot of an array row is the angular reflectance profile measured in a point along the fluidic channel (green curve in Fig. 1(e)). The starting frame fixes the reference vector $\left(p_{m}\right)_{0}$, where $p_{m}$ is the BSW resonance angular position along the ordinate $m$, both in pixels. For each successive frame $f$, we evaluated $\left(p_{m}\right)_{f}$ and the shift $\left(\Delta p_{m}\right)_{f}=\left(p_{m}\right)_{f}-\left(p_{m}\right)_{0}$, which was converted to $\Delta \theta(x, t)$ by means of the factors $c_{\theta}, c_{l}$ and the framerate. 
Then, $\Delta \theta(x, t)$ was converted to the local change of the effective refractive index of the fluid $\Delta n_{e f f}(x, t)$ making use of the volume sensitivity $S_{\mathrm{V}}$ of the sensor:

$$
\Delta n_{e f f}(x, t)=\Delta \theta(x, t) / S_{V},
$$

where $S_{V}=\partial \theta / \partial n=34 \mathrm{deg} / \mathrm{RIU}$ was measured by means of calibration experiments with liquids with known refractive index [2] under stationary conditions. $\Delta n_{e f f}(x, t)$ is the weighed effective index of the fluid in the layer probed by the BSW evanescent tail.

In Fig. 2, as an example, we plot the $\left|\Delta n_{\text {eff }}(\mathrm{x}, \mathrm{t})\right|$ signal retrieved by temporally monitoring the BSW resonance in two positions A and B (Fig. 1(d)) along the flow direction $\mathrm{x}$, separated by $\Delta x=3.24 \mathrm{~mm}$, when $\Phi=2.266 \mu \mathrm{L} / \mathrm{s}$.

As it can be seen from Fig. 2, a temporal delay between the two signals can be resolved. Since A was closer to the channel inlet, the refractive index increase was observed earlier than in B. We can evaluate the distance from the 1DPC surface $\xi(x, t)$ of the interface between the two solutions by means of the following expression [2]:

$$
\xi(x, t)=L_{p} \cdot \ln \frac{\Delta n_{M A X}}{\left|\Delta n_{e f f}(x, t)\right|},
$$

where $\Delta n_{M A X}=n_{s}-n_{0}$ is the refractive index difference between the solution and the pure DI water, respectively.

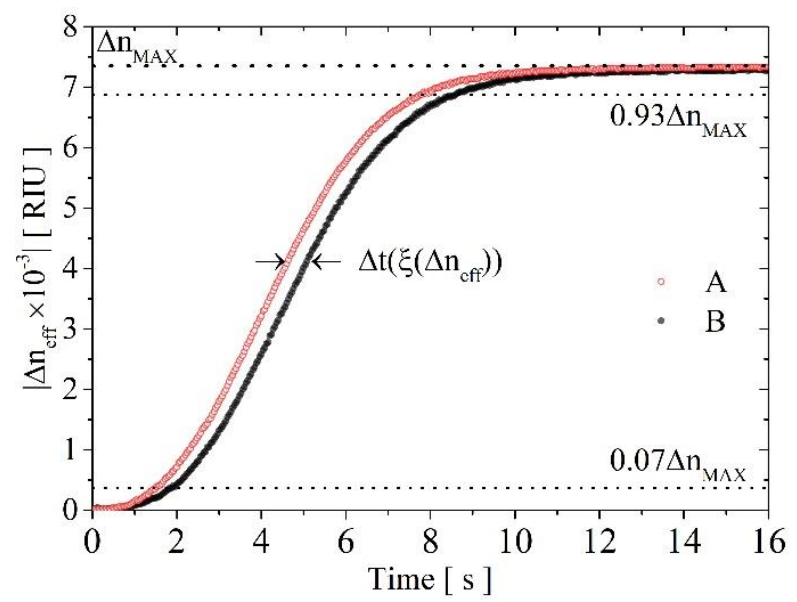

Fig. 2. Case $\Phi=2.266 \mu \mathrm{L} / \mathrm{s}$. Time dependency of the effective refractive index change in the A and B points shown in Fig. 1(d). The two dotted lines mark the interval inside which $v(\xi)$ was calculated via the Eq. (4).

To evaluate, under stationary conditions, the translation velocity of the contact line $v(\xi)$ as a function of the distance $\xi$ from the 1DPC surface, we extract, as shown in Fig. 2, the relative temporal delay between $\mathrm{A}$ and $\mathrm{B}, \Delta t\left(\Delta n_{e f f}\right)$, for each value of $\Delta n_{\text {eff }}$ and, correspondingly, of $\xi$. The velocity of the contact line as a function of $\xi$ is then given by:

$$
v(\xi)=\frac{\Delta x}{\Delta \mathrm{t}(\xi)}
$$

However, for the used $\mathrm{f}_{\mathrm{sam}}$, such a procedure gives rise to very noisy data for $\Delta n_{\text {eff }}$ and $\xi$ values corresponding to the flat portions of the curves shown in Fig. 2. It is therefore necessary to limit the procedure to a convenient interval of $\Delta n_{e f f}$, which limits the dynamic range for the measurement of $\xi(x, t)$. For the case shown in Fig. 2 ( $\Phi=2.266 \mu \mathrm{L} / \mathrm{s}), \Delta n_{e f f}$ is analyzed between $7 \%$ and $93 \%$ of $\Delta n_{M A X}$, corresponding to $\xi$ values between $\xi_{\min }=7 \mathrm{~nm}$ and $\xi_{\max }=280 \mathrm{~nm}$. For different $\Phi$ such an interval might have a slightly different width, depending on the quality of the signals. We point out that such a restriction is stronger than that imposed by the resolution of the technique for the measurement of the refractive index change, which is $\Delta n_{e f f, \min }=3 \times 10^{-6} \mathrm{RIU}$ [2]. The main limit to the accuracy on $\xi$ comes from the maximum available CMOS $f_{\text {sam. }}$.

The dynamic range $\left(\xi_{\max }\right)$ can be extended by increasing the penetration of the BSW evanescent tail inside the liquid. This can be achieved by increasing $\lambda_{0}$ to excite a BSW closer the TIR edge $[7,19]$. Alternatively, for fixed $\lambda_{0}$, one could modify the 1DPC geometry. However, this would also increase $\xi_{\text {min }}$ and deteriorate the accuracy. The accuracy could be recovered by either using a camera with larger $\mathrm{f}_{\text {sam }}$ or by increasing $\Delta n_{M A X}$. The contact line velocity curves retrieved from the measurements are plotted in Fig. 3. For each value of $\Phi$ used in the experiments, we plot $v_{N}=v(\xi) \cdot \Sigma / \Phi$, i.e. the velocity normalized to the average velocity across the channel. The $v_{N}$ curves are the average of repeated measurements: ten for $\Phi=4.058,3.162,2.266,1.370 \mu \mathrm{L} / \mathrm{s}$; four for $\Phi=0.474 \mu \mathrm{L} / \mathrm{s}$; two for $\Phi=0.365 \mu \mathrm{L} / \mathrm{s}$. The error bands amplitude is the standard deviation of the averages.

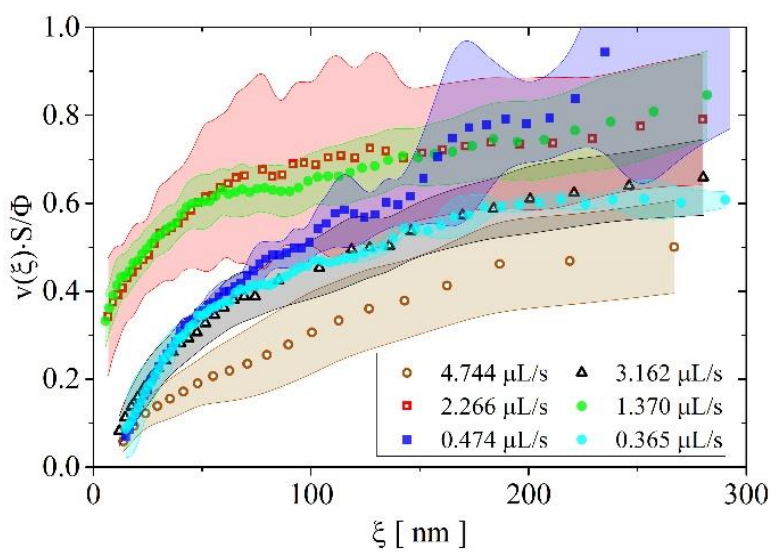

Fig. 3. Contact line velocity profiles measured for each flow rate $\Phi$. The curves are the average of ten $(4.058,3.162,2.266,1.370 \mu \mathrm{L} / \mathrm{s})$, four $(0.474 \mu \mathrm{L} / \mathrm{s})$ and two $(0.365 \mu \mathrm{L} / \mathrm{s})$ different experiments.

The plots appear qualitatively different over the investigated range of $\Phi$. Those obtained for the two lowest (blue, light blue) $\Phi$ show that, within the explorable $\xi$ interval, $v_{N}(\xi)$ decreases when approaching the boundary $(\xi=0)$. Even with the limitation set by $\xi_{m i n}$, it is likely that $v_{N}(\xi)$ gets zero at the boundary, suggesting the presence of a still boundary layer. With increasing $\Phi$ (red, green) $v_{N}(\xi)$ gets larger in proximity of the boundary and is still much different from zero, about $1 / 3$ of the average speed in the channel, at $\xi_{\min }=7 \mathrm{~nm}$. The limitation due to $\xi_{\min }$ does not permit to say whether a slipping layer is formed or not at the boundary. Further increasing $\Phi$, we observe a fall of $v_{N}(\xi)$ back to the behavior observed for small $\Phi$.

The results shown in Fig. 3 are qualitatively consistent with the theoretical model developed by Ledesma-Aguilar et al. $[26,27]$, which studied the slip velocity arising from diffusion effects near the contact line. The slip velocity in the vicinity of a solid boundary arises naturally in diffuse interface models, which consist of the usual Navier - Stokes equations coupled to a convection - diffusion equation. In their model the 
difference between the slip and average velocities determines whether the interface advances as a meniscus low and intermediate $\Phi$ ) or a thin layer of fluid is left adhered to the boundary wall (large $\Phi$ ). Moreover, in the limit for the solute diffusion coefficient close to zero, the model will reduce to the only Navier - Stokes equations which characterize the fluid velocity into the microchannel, and the Poiseuille equation represents a simple analytical solution in the case of a laminar regime $[3,26]$. At the present stage, we are not yet able to perform a quantitative comparison between the theoretical description and the results shown in Fig. 3, which will be addressed in our future work.

In conclusion, in this work, we developed an optical system based on BSWs to evaluate the contact line speed of a fluid flow in the low Reynold number condition in proximity of a micro-channel wall. The most important feature of the developed system is that the measurement of the contact line velocity at different distances from the boundary wall is carried out with a nanometric resolution. Within the limit imposed by diffusion of chemical species in the liquids, we think that the technique could be useful for velocimetry measurements at distance from surface not accessible by other techniques, such as the $\mu$-PIV [16] for example.

Funding. This work was partially funded by the European Commission funded project BILOBA (318035) and the Regione Lazio (Italy), Programme "Progetti di Gruppi di Ricerca”, funded project TURNOFF (85-2017-14945).

\section{REFERENCES}

[1] A. Manz, N. Graber and H.M. Widmer, Sens. Act. B Chem. 1, 244 (1990).

[2] A. Occhicone, A. Sinibaldi, F. Sonntag, P. Munzert, N. Danz and F. Michelotti, Sens. Act. B: Chem. 247, 532 (2017).

[3] C.C.L. Loureiro, A.M.N. Lima and H. Neff, J. of Phys.: conference series 85, (2007).

[4] P. Yeh, A. Yariv and C-S. Hon, J. Opt. Soc. Am. 67, 423 (1977).

[5] A.P. Vinogradov, A.V. Dorofeenko, A.M. Merzlikin and A.A. Lisyansky, Physics 53, 243, (2010).

[6] A. Sinibaldi, A. Fieramosca, R. Rizzo, A. Anopchenko, N. Danz, P. Munzert, C. Magistris, C. Barolo and F. Michelotti, Opt. Lett. 39, 2947 (2014).

[7] V.N. Konopsky and E.V. Alieva, Anal. Chem. 79, 4729 (2007).
[8] A. Sinibaldi, C. Sampaoli, N. Danz, P. Munzert, L. Sibilio, F. Sonntag, A. Occhicone, E. Falvo, E. Tremante, P. Giacomini and F. Michelotti, Biosens. Bioelectron. 92, 125 (2017).

[9] Y. Wan, Z. Zheng, W. Kong, X. Zhao, Y. Liu, Y. Bian and J. Liu, Opt. Exp. 20, 8998 (2012).

[10] S. Pirotta, X.G. Xu, A. Delfan, S. Mysore, S. Maiti, G. Dacarro, M. Patrini, M. Galli, G. Guizzetti, D. Bajoni, J.E. Sipe, G.C. Walker and M. Liscidini, J. Phys. Chem. C 117, 6821 (2013).

[11] G. Lerario, A. Cannavale, D. Ballarini, L. Dominici, M. De Giorgi, M. Liscidini, D. Gerace, D. Sanvitto, G. Gigli, Opt. Lett. 39, 2068 (2014).

[12] R. Dubey, B. Vosoughi Lahjani, E. Bakarat, M. Hayrinen, M. Roussey, M. Kuittinen, H. P. Herzig, Opt. Lett. 41, 4867 (2016).

[13] S.T. Wereley, and C.D. Meinhart, Annu. Rev. Fluid Mech. 42, 557 (2010).

[14] J. Westerweel, G.E. Elsinga, and A.J. Ronald, Annu. Rev. Fluid Mech. 45, 409 (2013).

[15] F. Scarano, Meas. Sci. Technol. 24, 012001 (2013).

[16] C. Zettner and M. Yoda, Exp. Fluids 34, 115 (2003).

[17] A. Zöller, R. Götzelmann, H. Hagedorn, W. Kiug, and K. Mati, SPIE 3133, 196 (1997).

[18] A. Sinibaldi, N. Danz, E. Descrovi, P. Munzert, U. Schulz, F. Sonntag, L. Dominici and F. Michelotti, Sens. Act. B: Chem. 174, 292 (2012).

[19] R. Rizzo, N. Danz, F. Michelotti, E. Maillart, A. Anopchenko and C. Wächter, Opt. Exp. 22, 23202 (2014).

[20] F. Michelotti, R. Rizzo, A. Sinibaldi, P. Munzert, C. Wächter, and N. Danz, Opt. Lett. 42, 2798 (2017).

[21] A. Sinibaldi, R. Rizzo, G. Figliozzi, E. Descrovi, N. Danz, P. Munzert, A. Anopchenko and F. Michelotti, Opt. Exp. 21, 23331 (2013).

[22] H. Raether, Surface Plasmons on Smooth and Rough surfaces and on Gratings, Berlin, Springer (1986).

[23] R. Wang, Y. Wang, D. Zhang, G. Si, L. Zhu, L. Du, S. Kou, R. Badugu, M. Rosenfeld, J. Lin, P. Wang, H. Ming, X. Yuan, and J.R. Lakowicz, ACS Nano 11, 5383 (2017).

[24] D.Zhang, R. Wang, Y. Xiang, Y. Kuai, C. Kuang, R. Badugu, Y. Xu, P. Wang, H. Ming, X. Liu, and J.R. Lakowicz, ACS Nano 11, 10446 (2017).

[25] T. M. Squires e S. R. Quake, Rev. Mod. Phys. 77, 977 (2005).

[26] R. Ledesma-Aguilar, A. Hernandez-Machado and I. Pagonabarraga, Phys. of Fluids 19, 102112 (2007).

[27] R. Ledesma-Aguilara, I. Pagonabarraga, and A. HernándezMachado, Phys. Fluids 19, 102113 (2007). 
[1] A. Manz, N. Graber and H.M. Widmer, «Miniaturized total chemical analysis systems: A novel concept for chemical sensing,» Sens. Act. B Chem., vol. 1, pp. 244-248, 1990.

[2] A. Occhicone, A. Sinibaldi, F. Sonntag, P. Munzert, N. Danz and F. Michelotti, «A novel technique based on Bloch surface waves sustained by one-dimensional photonic crystals to probe mass transport in a microfluidic channel,» Sens. Act. B: Chem., vol. 247, pp. 532-539, 2017.

[3] C.C.L. Loureiro, A.M.N. Lima and H. Neff, «Optical monitoring of micro fluidic flow conditions, employing surface plasmon resonance sensing,» J. of Phys.: conference series 85, 2007.

[4] P. Yeh, A. Yariv and C-S. Hon, «Electromagnetic propagation in periodic stratified media. I. General theory,» J. Opt. Soc. Am., vol. 67, n. 4, pp. 423-438, 1977.

[5] A.P. Vinogradov, A.V. Dorofeenko, A.M. Merzlikin and A.A. Lisyansky, «Surface states in photonic crystals,» Physics, vol. 53, n. 3, pp. 243-256, 2010.

[6] A. Sinibaldi, A. Fieramosca, R. Rizzo, A. Anopchenko, N. Danz, P. Munzert, C. Magistris, C. Barolo and F. Michelotti, «Combining label-free and fluorescence operation of Bloch surface wave optical sensors,» Opt. Lett., vol. 39, n. 10, pp. 29472950,2014

[7] V.N. Konopsky and E.V. Alieva, «Photonic crystal surface waves for optical biosensors,» Anal. Chem., vol. 79, n. 12, pp. 47294735, 2007.

[8] A. Sinibaldi, C. Sampaoli, N. Danz, P. Munzert, L. Sibilio, F. Sonntag, A. Occhicone, E. Falvo, E. Tremante, P. Giacomini and F. Michelotti, «Detection of soluble ERBB2 in breast cancer cell lysates using a combined label-free/fluorescence platform based on Bloch surface waves,» Biosens. Bioelectron., vol. 92, pp. 125130, 2017.

[9] Y. Wan, Z. Zheng, W. Kong, X. Zhao, Y. Liu, Y. Bian and J. Liu, «Nearly three orders of magnitude enhancement of GoosHanchen shift by exciting Bloch surface wave,» Opt. Exp., vol. 20, n. 8, pp. 8998-9003, 2012.

[10] S. Pirotta, X.G. Xu, A. Delfan, S. Mysore, S. Maiti, G. Dacarro, M. Patrini, M. Galli, G. Guizzetti, D. Bajoni, J.E. Sipe, G.C Walker and M. Liscidini, «Surface-Enhanced Raman Scattering in Purely Dielectric Structures via Bloch Surface Waves,» J. Phys. Chem. C, vol. 117, n. 13, p. 6821-6825, 21 March 2013.

[11] G. Lerario, A. Cannavale, D. Ballarini, L. Dominici, M. De Giorgi, M. Liscidini, D. Gerace, D. Sanvitto, G. Gigli, «Room temperature Bloch surface wave polaritons,» Opt. Lett., vol. 39, n. 7, pp. 2068-2071, 2014.

[12] R. Dubey, B. Vosoughi Lahjani, E. Bakarat, M. Hayrinen, M. Roussey, M. Kuittinen, H. P. Herzig, «Near-field characterization of a Bloch-surface-wave-based 2D disk resonator,» Opt. Lett., vol. 41, n. 21, pp. 4867-4870, 2016.

[13] S.T. Wereley, and C.D. Meinhart, «Recent Advances in MicroParticle Image Velocimetry,» Annu. Rev. Fluid Mech., vol. 42, pp. 557-576, 2010.
[14] J. Westerweel, G.E. Elsinga, and A.J. Ronald, «Particle Image Velocimetry for Complex and Turbulent Flows,» Annu. Rev. Fluid Mech., vol. 45, pp. 409-436, 2013.

[15] F. Scarano, «Tomographic PIV: principles and practice,» Meas. Sci. Technol., vol. 24, p. 012001, 2013.

[16] C. Zettner and M. Yoda, «Particle velocity field measurements in a near-wall flow using evanescent wave illumination,» Exp. Fluids, vol. 34, n. 1, pp. 115-121, 2003.

[17] A. Zöller, R. Götzelmann, H. Hagedorn, W. Kiug, and K. Mati, «Plasma ion assisted deposition: a powerful technology for the production,» SPIE, vol. 3133, pp. 196-204, 1997.

[18] A. Sinibaldi, N. Danz, E. Descrovi, P. Munzert, U. Schulz, F. Sonntag, L. Dominici and F. Michelotti, «Direct comparison of the performance of Bloch surface wave and surface plasmon polariton sensors,» Sens. Act. B: Chem., vol. 174, pp. 292-298, November 2012.

[19] R. Rizzo, N. Danz, F. Michelotti, E. Maillart, A. Anopchenko and C. Wächter, «Optimization of angularly resolved Bloch surface wave biosensors,» Opt. Exp., vol. 22, n. 19, pp. 23202-23214, 2014.

[20] F. Michelotti, R. Rizzo, A. Sinibaldi, P. Munzert, C. Wächter, and N. Danz, «Design rules for combined label-free and fluorescence Bloch surface wave biosensors,» Opt. Lett., vol. 42, n. 14, pp. 2798-2801, 2017.

[21] A. Sinibaldi, R. Rizzo, G. Figliozzi, E. Descrovi, N. Danz, P. Munzert, A. Anopchenko and F. Michelotti, «A full ellipsometric approach to optical sensing with Bloch surface waves on photonic crystals,» Opt. Exp., vol. 21, n. 20, pp. 23331-23344, 2013.

[22] H. Raether, Surface Plasmons on Smooth and Rough surfaces and on Gratings, Berlin: Springer, 1986.

[23] R. Wang, Y. Wang, D. Zhang, G. Si, L. Zhu, L. Du, S. Kou, R. Badugu, M. Rosenfeld, J. Lin, P. Wang, H. Ming, X. Yuan, and J.R. Lakowicz, «Diffraction-Free Bloch Surface Waves,» ACS Nano, vol. 11, n. 6, p. 5383-5390, 2017.

[24] D. Zhang, R. Wang, Y. Xiang, Y. Kuai, C. Kuang, R. Badugu, Y. $\mathrm{Xu}$, P. Wang, H. Ming, X. Liu, and J.R. Lakowicz, «Silver Nanowires for Reconfigurable Bloch Surface Waves,» ACS Nano, vol. 11, n. 10, p. 10446-10451, 2017.

[25] T. M. Squires e S. R. Quake, «Microfluidics: Fluid physics at the nanoliter scale,» Rev. Mod. Phys., vol. 77, pp. 977-1026, July 2005.

[26] R. Ledesma-Aguilar, A. Hernandez-Machado and I. Pagonabarraga, «Three-dimensional aspects of fluid flow in channels. I. Meniscus and thin film regimes,» Phys. of Fluids, vol. 19, p. 102112, 2007.

[27] R. Ledesma-Aguilara, I. Pagonabarraga, and A. HernándezMachado, «Three-dimensional aspects of fluid flows in channels. II. Effects of meniscus and thin film regimes on viscous fingers,» Phys. Fluids, vol. 19, n. 10, p. 102113, 2007. 